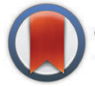

CrossMark \& click for updates

Cite this: Phys. Chem. Chem. Phys. 2015, 17, 20725

Received 15th May 2015, Accepted 13th July 2015

DOI: $10.1039 / c 5 c p 02818 c$

www.rsc.org/pccp

\section{Fluorescence switching of sanguinarine in micellar environments $\uparrow$}

\author{
Sagar Satpathi, Krishna Gavvala and Partha Hazra*
}

Sanguinarine (SANG), a key member of the benzylisoquinoline alkaloid family, is well-known for its various therapeutic applications such as antimicrobial, antitumor, anticancer, antifungal and anti-inflammatory etc. Depending on the medium $\mathrm{pH}$, SANG exists in the iminium or alkanolamine form, which emits at $580 \mathrm{~nm}$ and $420 \mathrm{~nm}$, respectively. Nucleophilic attack on the $C_{6}$ carbon atom converts the iminium form to the alkanolamine form of SANG, and these two forms are equally important for the medicinal activities of SANG. To improve its potency as a drug, it is essential to get a physical insight into this conversion process. In this study, we have deployed steady sate and time-resolved spectroscopic techniques to probe this conversion process inside different micellar environments. We have observed that the conversion from the iminium to alkanolamine form takes place in neutral OBG (octyl- $\beta$-D-glucopyranoside) and positively charged CTAB micelles, whereas the iminium form exclusively exists in negatively charged SDS micelles. This conversion from the iminium to alkanolamine form in the case of OBG and CTAB micelles may be attributed to the reduced $\mathrm{p} K_{\mathrm{a}}$ of this conversion process owing to the enhanced hydrophobicity experienced by the iminium form in between the surfactant head groups. On the other hand, the electrostatic attraction between positively charged iminium and negatively charged surfactant head groups stabilizes the iminium form in the stern layer of the SDS micelle. We believe that our observations are useful for selective transportation of any particular form of the drug into the active site. Moreover, loading of any particular form of drug can be easily monitored with the help of fluorescence color switch from orange (iminium) to violet (alkanolamine) without pursuing any sophisticated or complex technique.

\section{Introduction}

For several years, naturally occurring alkaloids have been receiving tremendous importance due to their potential pharmaceutical applications. Among them isoquinoline derivatives established themselves as one of the foremost alkaloids owing to their important contribution in biomedical research. ${ }^{1}$ Being a representative of the benzylisoquinoline alkaloid family, ${ }^{2,3}$ sanguinarine (SANG) exhibits numerous important biological activities such as anti-inflammatory, antioxidant, antifungal and antimicrobial etc. ${ }^{4-7}$ Recent studies reveal that SANG can instigate apoptosis in various cancer cells. ${ }^{8}$ Not only by inducing apoptosis, but it can also exhibit anticancer activity by means of inhibiting telomerase and topoisomerase enzyme activity. ${ }^{9,10}$ Thus, SANG can act as a G-quadruplex stabilizer and hence it prospects as a potential anticancer drug. ${ }^{10}$ Due to its diverse application in biomedical research, some attention has been paid to understand the

Department of Chemistry, Indian Institute of Science Education and Research (IISER), Pune, Pune (411008), Maharashtra, India. E-mail: p.hazra@iiserpune.ac.in; Fax: +91-20-2589-9790; Tel: +91-20-2590-8077

$\dagger$ Electronic supplementary information (ESI) available. See DOI: 10.1039/ c5cp02818c interactions of SANG with different biomolecules like proteins, polymorphic nucleic acid structures including DNA (B form, $\mathrm{Z}$ form, triplex, G-quadruplex) and RNA etc. ${ }^{10-16}$ SANG generally exists in two forms (Scheme 1) namely the cationic form (i.e. iminium form) at lower $\mathrm{pH}(\leq 6.5)$ and the neutral form (i.e. alkanolamine form or pseudobase form) at higher $\mathrm{pH}(\geq 7.2) \cdot{ }^{17,18}$ The iminium form is unsaturated and completely planar; whereas the alkanolamine form is non-planar. The conversion from iminium to alkanolamine takes place due to nucleophilic attack of water/hydroxide ion on the $\mathrm{C}_{6}$ carbon atom, which is electrophilic in nature due to the presence of an adjacent quaternary nitrogen atom. ${ }^{19}$ The equilibrium between the iminium and alkanolamine form is largely dependent on the $\mathrm{pH}$ of the medium. ${ }^{17}$ Several biomolecules have different affinity for these

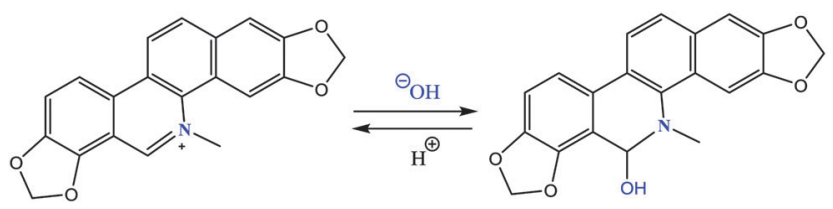

Scheme 1 Chemical structures of iminium and alkanolamine forms of sanguinarine. 
two forms of SANG. The iminium form was found to be the predominant form for binding with nucleic acids, ${ }^{16,20}$ whereas, exactly opposite binding preference has been observed for interaction with serum protein i.e. the alkanolamine form being the favored one. ${ }^{21}$ The most unique property of SANG is its photophysical property due to its distinctly different emission maximum position for the iminium $(580 \mathrm{~nm})$ and alkanolamine form $(420 \mathrm{~nm}) .{ }^{17}$ The huge difference $(\sim 150 \mathrm{~nm})$ in emission maximum between these two forms makes it very useful for probing the abasic site in DNA. Thus, SANG has been employed to find the abasic site in DNA. This shows that the iminium form is preferred over the alkanolamine form in the DNA abasic site, which is evident from their characteristic change in fluorescence peaks. $^{22}$ Likewise the DNA abasic site, metalloprotein hemoglobin, was also found to have preference for the iminium form. ${ }^{23}$ Not only with biomolecules, SANG has also been studied in drug delivery vehicles like cucurbituril and cyclodextrin nano-cavities considering its potential usefulness in biomedical research. It was found that inside both the nano-cavities due to inhibition of nucleophilic attack the iminium form is stabilized. ${ }^{18,24}$ In order to put forward SANG as a potential drug, it is very important to explore the pharmacokinetic and pharmacodynamic behavior of the SANG, which will give a better insight about the characteristic properties of the drugs i.e. ADME (absorption, distribution, metabolism, and excretion). To monitor these characteristics, micelles are considered to be the simplest and useful model due to their eco-friendly nature and tunable properties. ${ }^{25,26}$

Most of the biological systems in nature are highly anisotropic and restricted in nature. Inspired from nature, researchers have invested a reasonable amount of time in understanding the photophysical properties of drugs inside biomimicking environments. ${ }^{27-31}$ These biomimicking systems include self-organized arrangements like micelles, reverse micelles, vesicles and molecular containers (i.e. cucurbituril, cyclodextrin etc.). ${ }^{18,24,31-33}$ Among them, micelles are considered to be one of the simplest ordered assemblies due to their highly cooperative, thermodynamically stable structure. Therefore, micelles have gained tremendous interest for their potential application in diverse fields such as drug delivery vehicles, in energy storage and as electronic devices. ${ }^{34-37}$ Micelles have been widely utilized as drug carriers owing to their ability to enhance the solubility, stability and bio-availability of the drug by the incorporation of the drug in micellar environments. ${ }^{25,26}$ Thus, it is necessary to understand the effect of micellar confinement on the solubility and stability of SANG in order to use it in the pharmaceutical field. Moreover, to understand how SANG is transported to its target, it is very important to establish a relationship between the environment and the photophysical properties of SANG. In the present study, we have made an attempt to obtain the microenvironment alteration and photophysics modulation of SANG inside different types of micellar environments using steady state and timeresolved spectroscopy techniques. Interestingly, we have noticed that cationic and neutral micelles (i.e. CTAB and OBG) trigger the conversion from the iminium to alkanolamine form, whereas the iminium form is stabilized in the anionic micelle (SDS). Moreover, our results suggest that this conversion from the iminium to alkanolamine form can be easily monitored with the help of fluorescence switch from orange to blue/violet color. We believe that our results might provide new insight towards the improvement of pharmaceutical applications of sanguinarine.

\section{Experimental section}

Sanguinarine (SANG), octyl- $\beta$-D-glucopyranoside (OBG), sodium dodecyl sulfate (SDS), and cetyltrimethylammonium bromide (CTAB) were purchased from Sigma Aldrich, and used without further purification. Millipore water $(\mathrm{pH}$ 6.5) was used for sample preparation. Surfactants were gradually added to the solution containing SANG, and the solution was gently shaken after each addition of surfactant until complete solubilization took place. Moreover, we have given 20 minutes equilibration time for each addition of surfactant. A buffer solution of $\mathrm{pH} 9$ has been prepared using Tris- $\mathrm{HCl}$ salt.

Absorbance measurements were performed on a Shimadzu UV-visible spectrophotometer (UV-2600), and steady-state fluorescence spectra were recorded using a FluoroMax-4 spectrofluorimeter (Horiba Scientific, USA). All time-resolved fluorescence measurements (both life-time as well as anisotropy) were taken on a time correlated single photon counting (TCSPC) spectrometer (Horiba Jobin Yvon IBH, U.K.). The detailed description of the instrument is described elsewhere. ${ }^{38,39}$ Briefly, excitation of drug molecules has been done using $340 \mathrm{~nm}$ nano-led (pulse width $\sim 1 \mathrm{~ns}$ ) and $375 \mathrm{~nm}$ (pulse width $<200 \mathrm{ps}$ ) diode lasers. For anisotropy $(r(t))$ measurements, the emission polarization was adjusted to be parallel or perpendicular to that of the excitation, and anisotropy is defined as

$$
r(t)=\frac{I_{\|}(t)-G I_{\perp}(t)}{I_{\|}(t)+2 G I_{\perp}(t)}
$$

where $I_{\|}(t)$ and $I_{\perp}(t)$ are fluorescence decays polarized parallel and perpendicular to the polarization of the excitation light, respectively. $G$ is the correction factor for detector sensitivity to the polarization direction of the emission and the measured $G$ values are 0.5 and 0.62 for $340 \mathrm{~nm}$ and $375 \mathrm{~nm}$ excitation wavelength, respectively. $G$ factor was measured using horizontally polarization excitation light and collecting the horizontal $\left(I_{\mathrm{HH}}(t)\right)$ and vertical $\left(I_{\mathrm{HV}}(t)\right)$ decay profiles of emitted light. The analysis of lifetime and anisotropy data was done using the IBH DAS6 analysis software. We have fitted both lifetime as well as anisotropy data with a minimum number of exponentials. The quality of each fitting was judged by $\chi^{2}$ values and the visual inspection of the residuals. The value of $\chi^{2} \approx 1$ was considered as the best fit for the plots. We have calculated the quantum yield of the alkanolamine form and the iminium form at $\mathrm{pH} 6.5$ taking a standard quantum yield value for the alkanolamine and iminium form. ${ }^{22}$

\section{Results and discussion}

\section{Steady state study}

Generally SANG exists in two forms i.e. iminium and alkanolamine form (Scheme 1) and the conversion between these two 
forms is largely dependent on the $\mathrm{pH}$ of medium. The absorption spectrum of this iminium form offers four absorption bands in the UV-vis range with peaks at 275, 330, 396 and $475 \mathrm{~nm}$ which corresponds to the electronic $\pi \rightarrow \pi^{*}$ transition. But the same for alkanolamine exists only at 285 and $326 \mathrm{~nm}$ devoid of any band at higher wavelength. ${ }^{40}$ In CTAB and OBG micelles, the characteristic peaks for the iminium form (peaks at 396 and $475 \mathrm{~nm}$ ) are reduced indicating the lesser ground state population of the iminium form (Fig. S1b and c, ESI $\dagger$ ), whereas in the SDS micelle it exhibits a small red shift (396 and $475 \mathrm{~nm}$ peak) suggesting the stabilization of the iminium form of SANG (Fig. S1a, ESI $\dagger$ ). Steady state emission studies have been performed to get insight into the excited state behavior of sanguinarine (SANG) in different types of micellar environments (Fig. 1). The emission spectrum (excited at $320 \mathrm{~nm}$ ) of SANG in water $(\mathrm{pH} \sim 6.5)$ exhibits a dominating peak at $580 \mathrm{~nm}$ along with a peeping peak at $\sim 420 \mathrm{~nm}$ (Fig. 1 ). The lower energy peak is attributed to the iminium form of SANG, whereas the weak peak at $420 \mathrm{~nm}$ indicates the presence of the alkanolamine form of SANG. ${ }^{17,18}$ The presence of a minute population of the alkanolamine form is expected as the conversion from the iminium to alkanolamine form takes place at $\mathrm{pH} \sim 7.5 .{ }^{17,18}$ With gradual addition of SDS to the SANG containing water solution, emission maximum at higher wavelength (at $580 \mathrm{~nm}$ ) exhibits blue shift along with a slight increment in intensity up to the critical micelle concentration $(<8 \mathrm{mM})$. After CMC $(\geq 8 \mathrm{mM})$, the characteristics peak of the iminium form reappears at $580 \mathrm{~nm}$ and a small increase in intensity of the alkanolamine peak at $\sim 440 \mathrm{~nm}$ (Fig. 1a) is observed. The aberration in results below CMC $(<8 \mathrm{mM})$ may be due to the electrostatic interaction between the negatively charged SDS surfactant head group and the positively charged drug molecule (Fig. S2, ESI $\dagger$ ). Above CMC ( $\geq 8 \mathrm{mM}$ ), the effect of micelle formation predominates over this electrostatic interaction; thereby, it shows enhancement and recovery of the iminium form along with a slight increment in the alkanolamine peak intensity at $\sim 450 \mathrm{~nm}$. A similar type of enhancement is also observed for excitation spectra monitored at $580 \mathrm{~nm}$ (i.e. iminium form) which indicates the stabilization of the iminium form in the SDS micellar environment (Fig. S4, ESI $\dagger$ ). Noteworthy changes in fluorescence spectral features of SANG are observed in the presence of CTAB (Fig. 1b). Upon a gradual increase in the concentration of CTAB, the peak at $\sim 420 \mathrm{~nm}$ becomes prominent particularly after CMC. With a further increase in concentration, the intensity at $420 \mathrm{~nm}$ tremendously increases along with a blue shift; whereas the peak intensity at $580 \mathrm{~nm}$ systematically decreases, although it does not vanish even at a very high concentration of CTAB (Fig. 1b). Notably, at any concentration above CMC $(>8 \mathrm{mM})$, the intensity of the higher energy peak dominates over that of the lower energy peak. Similar changes in emission spectral features are observed in the case of the OBG micellar environment (Fig. 1c). However, the peak at $420 \mathrm{~nm}$ is more blue shifted in the OBG micellar environment compared to the CTAB micelle (Fig. 1c).

Notably, all the significant modulation in photophysical behavior of SANG comes into the picture above the respective CMC of surfactants. Thus, the changes in emission profiles
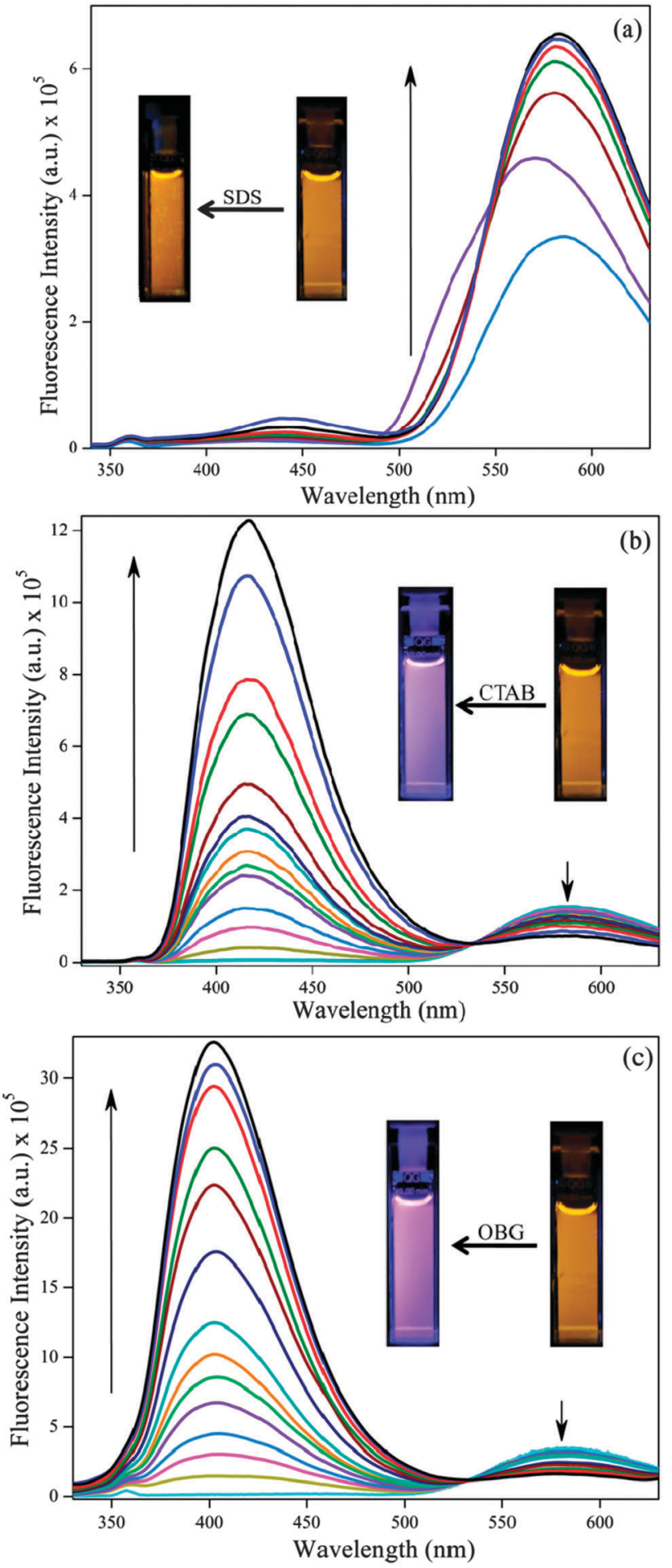

Fig. 1 Emission spectra of sanguinarine $(\sim 10 \mu \mathrm{M})$ with increasing concentration of different types of surfactants (a) SDS (0 to $41 \mathrm{mM})$, (b) CTAB (0 to $32.5 \mathrm{mM}$ ) and (c) OBG (0 to $163 \mathrm{mM}$ ). The direction of arrow indicates lower to higher concentration.

observed are attributed to the effect of entrapment of SANG inside micellar environments. The most striking observation of our results is the prominent appearance of the alkanolamine form of SANG in CTAB and OBG micelles. The observed increment in 
the higher energy peak in both micellar systems $(\sim 420 \mathrm{~nm})$ reflects the enhancement in alkanolamine population, and attenuation of the lower energy peak (580 nm) indicates the gradual decrement in the iminium form. In the presence of $\mathrm{CTAB}$ and the OBG micellar environment, the excitation spectrum monitored at $580 \mathrm{~nm}$ (i.e. iminium form) decreases and the same for the alkanolamine form (monitored at $420 \mathrm{~nm}$ ) shows a gradual enhancement (Fig. S5 and S6, ESI $\dagger$ ). These observations lead us to conclude that the above two micellar environments favor the conversion from the iminium to alkanolamine form of SANG. The tremendous increase in intensity of the alkanolamine form compared to the iminium form may be either due to the reduction of the non-radiative decay pathway and/or an increase in the radiative decay pathway of the alkanolamine form of the SANG inside the micellar environment. Moreover, it is reported that the fluorescence quantum yield of the alkanolamine form is significantly higher than that of the iminium form of SANG. ${ }^{17,22}$ The presence of an iso-emissive point at $530 \mathrm{~nm}$ indicates that both the iminium and alkanolamine forms are in equilibrium in the excited state. The more blue shifted alkanolamine peak may be appearing due to the less polar environment experienced by SANG in the presence of the OBG micellar environment compared to the CTAB micelle.

It is very well known that the drug/probe molecule inside the micelle can stay in three different locations, namely, Stern/ palisade (ionic micelle/neutral micelle) layer, in between the surfactant head groups and the hydrocarbon core of the micelle. ${ }^{41}$ Stern/palisade layers are comprised of polar head groups, counter ions (for ionic surfactants) and water molecules. Notably, the water molecules inside the Stern/palisade layer behave differently compared to bulk water. ${ }^{42}$ Even the dynamics of these water molecules in micellar environments are drastically retarded compared to bulk water medium. ${ }^{41}$ Positively charged SANG molecules (i.e. its iminium form) prefer to stay in the negatively charged Stern layer of SDS micelles due to the electrostatic interaction, which leads to the stabilization of this cationic form of the drug. In consequence, the emission peak at lower energy $(580 \mathrm{~nm})$ is enhanced with the gradual addition of SDS surfactant. Unlike SDS, the Stern layer of the CTAB micelle is positively charged, whereas the palisade layer of the OBG micelle is neutral. Thus, the positively charged SANG (i.e. iminium form) molecules do not experience any stabilization in the Stern/ palisade layer of these micelles (CTAB and OBG). Hence, the drug molecules try to stay away from the Stern/palisade layer and preferably reside in between the surfactant head groups. Here, it is pertinent to mention that the $\mathrm{p} K_{\mathrm{a}}$ of the probe is modulated by the hydrophobicity of the medium. ${ }^{43,44}$ A greater hydrophobicity in a micellar environment can decrease the $\mathrm{p} K_{\mathrm{a}}$ of the probe due to less polarity of the surrounding environment. ${ }^{43-45}$ Positively charged SANG (iminium form) molecules in between the surfactant head groups experience a reasonable hydrophobicity than that in bulk water medium. This makes the C6 carbon (adjacent to positively charged nitrogen atom) atom to be more electron deficient in nature. Thus, the conversion from the iminium to alkanolamine form becomes even more facile in these micellar environments (CTAB, OBG). Notably, it is reported that the $\mathrm{pH}$ of water molecules residing at the CTAB micellar surface is higher $(\mathrm{pH} \sim 9.5)$ than that of bulk water $(\mathrm{pH}=7) .{ }^{44,45}$ This may be another contributing factor for this conversion process in the CTAB micellar environment. ${ }^{45}$ Based on these facts, it is logical to predict that the $\mathrm{p} K_{\mathrm{a}}$ for the conversion from the iminium to alkanolamine form decreases in the presence of OBG and CTAB micellar environments. We have also calculated the percentage of molecules being converted from the iminium to alkanolamine form by monitoring the reduction in emission intensity at $580 \mathrm{~nm}$ by exciting the iminium form exclusively at $475 \mathrm{~nm}$ (Fig. S3, ESI $\dagger$ ). Our calculation suggests that the percentage of molecules converted from the iminium to alkanolamine form is $\sim 50 \%$ and $\sim 60 \%$ for OBG and CTAB micelles, respectively. In a nutshell, the steady state results of SANG in the presence of micellar environments manifest the fact that the conversion from the iminium to alkanolamine form can be probed in CTAB and OBG micelles, without increasing the $\mathrm{pH}$ of the medium.

\section{Time resolved study}

We have employed the time-resolved fluorescence measurement technique, which is an excellent tool to monitor the dynamics of molecules in the excited state, and is a sensitive technique in identifying multiple emissive species present in a system. Decay profiles of SANG in the absence and presence of micellar environments are collected at $\sim 400 \mathrm{~nm}$ (exciting by $340 \mathrm{~nm}$ nano-led) and $580 \mathrm{~nm}$ (exciting at $375 \mathrm{~nm}$ diode laser) to monitor the excited-state dynamics of alkanolamine and iminium forms, respectively. Fluorescence transients are shown in Fig. 2 and the results are presented in Table 1 . Iminium and alkanolamine forms in water exhibit a single exponential decay (collected at $580 \mathrm{~nm}$ and $415 \mathrm{~nm}$, respectively) with lifetimes of $2.37 \mathrm{~ns}$ and $3.16 \mathrm{~ns}$, respectively. In the case of the SDS micelle, the lifetime of the iminium form increases from 2.37 (in water) to $4.82 \mathrm{~ns}$ (Table 1). This observation confirms our claim that the positively charged iminium form residing at the Stern layer of the SDS micelle gets stabilized through electrostatic interaction with the negatively charged head group of the surfactant. This confinement of the iminium form inside the micellar environment reduces the non-radiative decay pathways to several fold, which accounts for the increment in excited state lifetime. In the case of the OBG micellar environment, the lifetime of the alkanolamine form (monitored at $420 \mathrm{~nm}$ ) increases, whereas the decrement in lifetime is observed in the CTAB micelle (Table 1). The increased lifetime in the OBG micelle may be attributed to the encapsulation of the drug in a confined environment. Moreover, the lifetime of the alkanolamine form reduces in the CTAB micelle. The above-mentioned lifetime results can be rationalized in terms of alternations in radiative and non-radiative decay pathways (eqn (2) and (3)) of SANG in the presence of confined micellar environments.

$$
\begin{gathered}
\phi_{\mathrm{f}}=k_{\mathrm{r}} \tau_{\mathrm{f}} \\
\frac{1}{\tau_{\mathrm{f}}}=k_{\mathrm{r}}+\sum k_{\mathrm{nr}}
\end{gathered}
$$



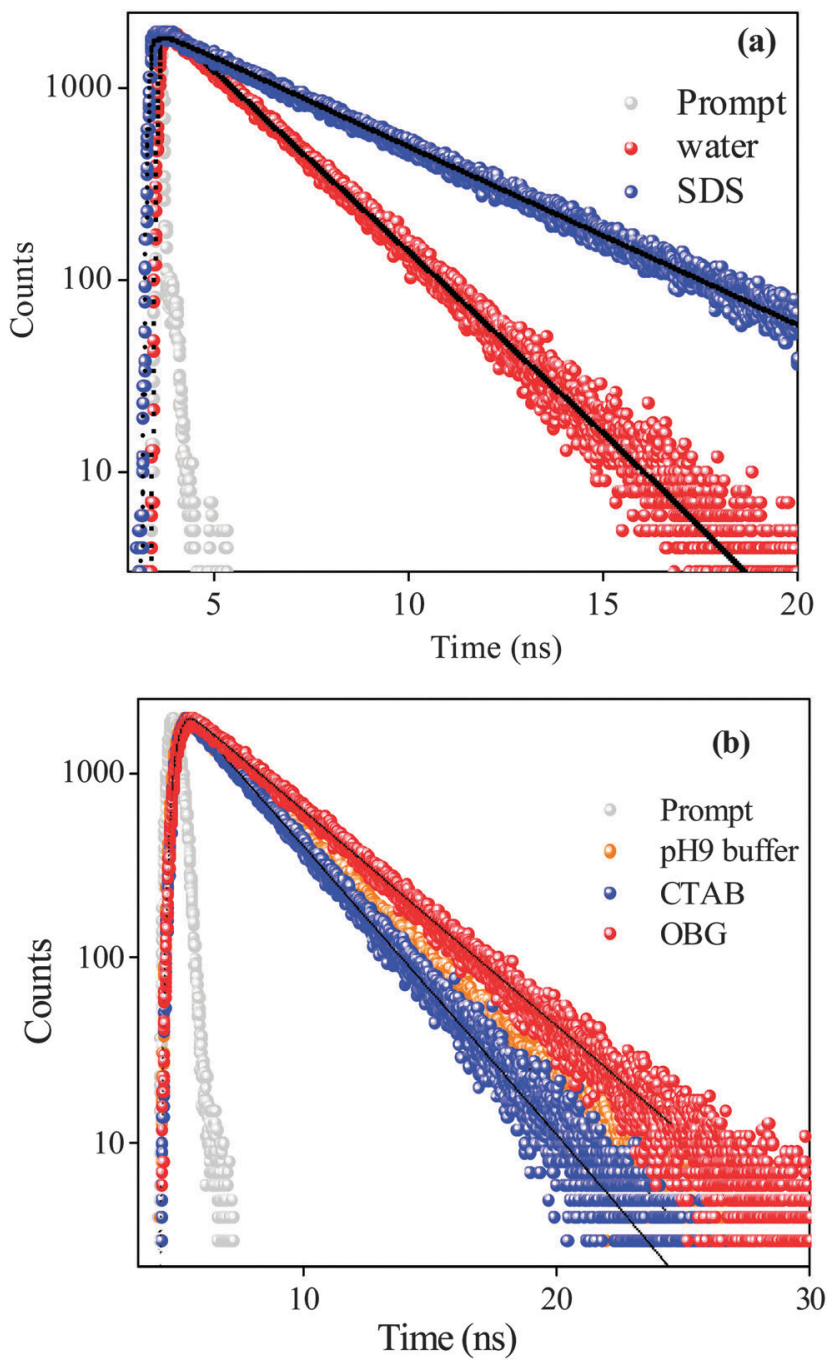

Fig. 2 Fluorescence transients of sanguinarine (a) in water and SDS $\left(\lambda_{\mathrm{ex}}=\right.$ $\left.375 \mathrm{~nm}, \lambda_{\mathrm{em}}=580 \mathrm{~nm}\right)$, (b) in $\mathrm{pH} 9$ buffer $\left(\lambda_{\mathrm{ex}}=340 \mathrm{~nm}, \lambda_{\mathrm{em}}=420 \mathrm{~nm}\right)$, $\operatorname{CTAB}\left(\lambda_{\mathrm{ex}}=340 \mathrm{~nm}, \lambda_{\mathrm{em}}=415 \mathrm{~nm}\right)$ and OBG $\left(\lambda_{\mathrm{ex}}=340 \mathrm{~nm}, \lambda_{\mathrm{em}}=403 \mathrm{~nm}\right)$ micelles.

Table 1 Fluorescence transient fittings of sanguinarine in water, buffer and different micellar environments

\begin{tabular}{llllll}
\hline Sample & $\lambda_{\text {ex }}(\mathrm{nm})$ & $\lambda_{\text {em }}(\mathrm{nm})$ & $a_{1}$ & $\tau_{1}(\mathrm{~ns})$ & $\chi^{2}$ \\
\hline SANG in water (pH 6.5) & 375 & 580 & 1 & 2.38 & 0.99 \\
SANG + 41 mM SDS & 375 & 580 & 1 & 4.82 & 1.09 \\
SANG + 163 mM OBG & 375 & 580 & 1 & 3.08 & 1.16 \\
SANG + 32.5 mM CTAB & 375 & 580 & 1 & 2.28 & 1.05 \\
SANG in buffer (pH 9.5) & 340 & 415 & 1 & 3.16 & 1.04 \\
SANG + 163 mM OBG & 340 & 403 & 1 & 3.85 & 1.04 \\
SANG + 32.5 mM CTAB & 340 & 415 & 1 & 2.88 & 1.05 \\
& & & & &
\end{tabular}

where $\phi_{\mathrm{f}}$ represents the quantum yield, $\tau_{\mathrm{f}}$ is the lifetime of the excited state species. $k_{\mathrm{r}}$ and $k_{\mathrm{nr}}$ are the radiative and nonradiative rate constants, respectively. We have calculated the quantum yields, and radiative and non-radiative constants for respective systems which are listed in Table 2 . Since the lifetime of the alkanolamine form is reduced (Table 1) and the intensity of the same form is enhanced in the presence of a CTAB
Table 2 Quantum yields, radiative and non-radiative constants of sanguinarine in water ( $\mathrm{pH}$ 6.2) and different micellar environments

\begin{tabular}{lllll}
\hline Sample & \multicolumn{3}{c}{$\begin{array}{l}k_{\mathrm{r}} \times 10^{12} \\
\left(\mathrm{~s}^{-1}\right)\end{array}$} & $\begin{array}{l}k_{\mathrm{nr}} \times 10^{12} \\
\left(\mathrm{~s}^{-1}\right)\end{array}$ \\
\hline $\begin{array}{l}\text { SANG in } \mathrm{H}_{2} \mathrm{O} \text { (iminium) } \\
\text { pH 6.5 }\end{array}$ & 0.00307 & 2.38 & 1.29 & 419 \\
SANG in SDS (iminium) & 0.00594 & 4.82 & 1.23 & 206 \\
$\begin{array}{l}\text { SANG in buffer (alkanolamine) } \\
\text { pH 9.5 }\end{array}$ & 0.0013 & 3.16 & 0.41 & 309 \\
SANG in OBG (alkanolamine) & 0.0683 & 3.85 & 17.7 & 240 \\
SANG in CTAB (alkanolamine) & 0.09 & 2.88 & 31 & 320 \\
& & & &
\end{tabular}

micellar environment (Fig. 2b), it suggests that the increase in the radiative rate constant $\left(k_{\mathrm{r}}\right)$ of the alkanolamine form $\left(0.41 \mathrm{ps}^{-1}\right.$ to $\left.31 \mathrm{ps}^{-1}\right)$ is significantly higher than the change in the non-radiative decay rate constant $\left(k_{\mathrm{nr}}\right)$ of the alkanolamine form. In fact the non-radiative decay rate constant $\left(k_{\mathrm{nr}}\right)$ of the alkanolamine form enhances in the presence of a CTAB micellar environment $\left(309 \mathrm{ps}^{-1}\right.$ to $\left.320 \mathrm{ps}^{-1}\right)$. But in the case of the OBG micellar environment, the non-radiative $\left(k_{\mathrm{nr}}\right)$ and radiative decay constant $\left(k_{\mathrm{nr}}\right)$ of the alkanolamine form is found to be decreased and increased, respectively, which is also evident from the respective changes in their quantum yield and excited state lifetime value (Table 2). This increment in non-radiative decay channels in CTAB micelles can be rationalized in terms of the heavy atom perturbation effect. ${ }^{46}$ The presence of bromide ions promotes the intersystem crossing phenomenon $\left(\mathrm{S}_{1} \rightarrow \mathrm{T}_{1}\right)$ via spin-orbit coupling. ${ }^{47}$ Thus, the excited state lifetime of the alkanolamine form decreases in the presence of a CTAB micellar environment due to the enhancement in non-radiative decay pathways. Similar kinds of enhancement in non-radiative decay pathways are also perceived for many organic compounds in CTAB micellar environments. ${ }^{48,49}$ The same effect is absent in OBG micelles, as the micellar surface is devoid of any heavy ions. Therefore, its lifetime increases due to the confinement effect in OBG micellar environments. In summary, the lifetime results are in harmony with the steady state results, which infers that the conversion from iminium to alkanolamine takes place inside OBG and CTAB micelles, whereas the iminium form gets stabilized in the SDS micelle.

Time-resolved anisotropy measurement has been employed to get an idea about the location as well as surrounding environments around the drug. The rotational relaxation time of SANG in water (pH 6.5) is $\sim 160 \mathrm{ps}$ (monitored at emission maxima of the iminium form at $580 \mathrm{~nm}$ upon excitation at $375 \mathrm{~nm}$ ). Anisotropy decay transients for SANG in different micellar environments show significant changes in the rotational relaxation time (Table 3, Fig. 3). In the case of OBG and CTAB micellar systems, the anisotropy decay profiles show the increment in average rotational relaxation time to the nanosecond time scale $(1.97 \mathrm{~ns}$ for CTAB and $2.30 \mathrm{~ns}$ for OBG micelle). This increase in rotational relaxation time is indicative of the confinement for SANG molecules inside CTAB and OBG micelles. In the case of the SDS micelle, the anisotropy transient (monitored at $580 \mathrm{~nm}$ ) fitting exhibits a rotational relaxation time of $\sim 750 \mathrm{ps}$, which is almost five times slower in comparison with the rotational relaxation 
Table 3 Time-resolved anisotropy decay parameters of sanguinarine in water, buffer and different micellar environments

\begin{tabular}{|c|c|c|c|c|c|c|c|c|c|}
\hline Sample & $\lambda_{\text {ex }}(\mathrm{nm})$ & $\lambda_{\mathrm{em}}(\mathrm{nm})$ & $\tau_{1}(\mathrm{~ns})$ & $a_{1}$ & $\tau_{2}(\mathrm{~ns})$ & $a_{2}$ & $\tau_{\mathrm{r}}(\mathrm{ns})$ & $D_{\mathrm{h}}(\AA)$ & $\chi^{2}$ \\
\hline SANG + $41 \mathrm{mM}$ SDS & 375 & 580 & 0.75 & 1 & - & - & 0.75 & 18.06 & 1.04 \\
\hline SANG + $163 \mathrm{mM}$ OBG & 340 & 404 & 0.36 & 0.10 & 2.50 & 0.90 & 2.30 & 26.24 & 1.04 \\
\hline SANG in pH 9.5 Buffer & 340 & 420 & 0.35 & 1 & - & - & 0.35 & - & 0.94 \\
\hline
\end{tabular}

time of SANG in water. More retarded rotational relaxation time in CTAB and OBG micelles than that of SDS micelle suggests that drug molecules located in more restricted environments, in the former two micelles, probably reside in between surfactant head groups; whereas in the SDS micelle, the drug molecules stay at the Stern layer exhibiting faster relaxation dynamics $(\sim 0.7 \mathrm{~ns})$ compared to other micellar environments. We have also determined the hydrodynamic diameter of the drug-micelle complex from the Stokes-Einstein relationship ${ }^{50}$ (Note S1, ESI $\dagger$ ). The obtained hydrodynamic diameter values for different micelles (Table 3) are in good agreement with literature values, ${ }^{51-53}$ which further validate the encapsulation of the drug in micellar environments. In summary, anisotropy results confirm that positively charged SANG molecules reside at the negatively charged Stern layer of the SDS micelle and get stabilized in this micellar environment, whereas SANG molecules encapsulated in between polar head groups (with pyridine nitrogen exposed towards the surface) experience reasonable hydrophobicity in OBG and CTAB micelles, and are majorly responsible for the change in $\mathrm{p} K_{\mathrm{a}}$ of drug molecules and subsequent conversion from the iminium to alkanolamine form of SANG.

SANG exhibits different binding modes with several supramolecular assemblies and biological nano-cavities. ${ }^{18,21,23,24}$ In biological nano-cavities (like serum protein, hemoglobin), preference for both the iminium and alkanolamine forms has been observed. The serum protein was found to have a more binding affinity for the alkanolamine form, ${ }^{21}$ whereas the exactly

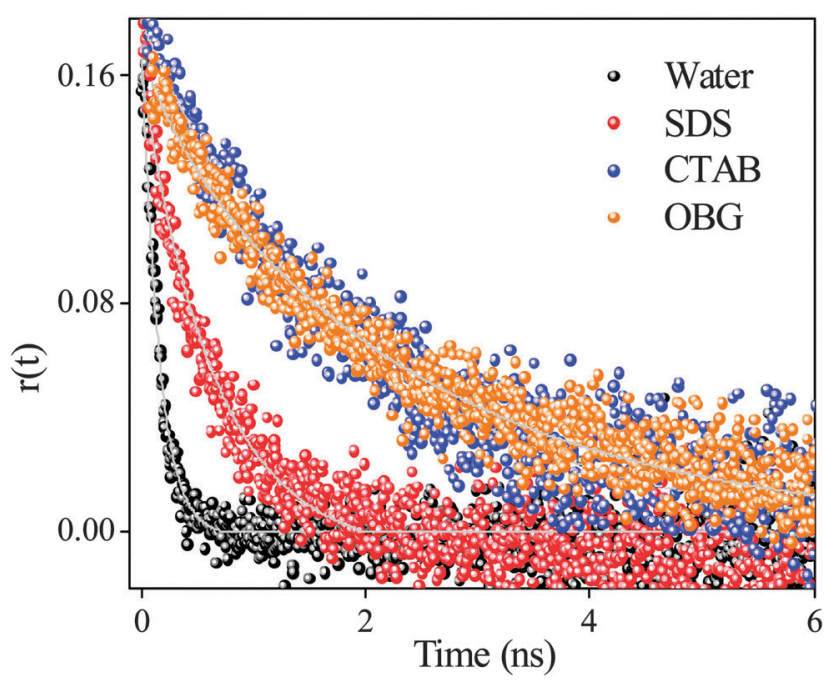

Fig. 3 Anisotropy decays of sanguinarine in water $\left(\lambda_{\mathrm{ex}}=375 \mathrm{~nm}, \lambda_{\mathrm{em}}=\right.$ $580 \mathrm{~nm}), \operatorname{CTAB}\left(\lambda_{\mathrm{ex}}=340 \mathrm{~nm}, \lambda_{\mathrm{em}}=415 \mathrm{~nm}\right), \mathrm{OBG}\left(\lambda_{\mathrm{ex}}=340 \mathrm{~nm}, \lambda_{\mathrm{em}}=\right.$ $404 \mathrm{~nm})$ and SDS $\left(\lambda_{\mathrm{ex}}=375 \mathrm{~nm}, \lambda_{\mathrm{em}}=580 \mathrm{~nm}\right)$ micelles. opposite binding preference has been observed for hemoglobin. ${ }^{23}$ Binding of the positively charged iminium form is majorly driven through the electrostatic interaction with the amino acid residue, whereas hydrophobicity plays the key role for the alkanolamine form. ${ }^{21,23}$ Biczók and co-workers reported the preferential encapsulation of the iminium form and the protection of the $\mathrm{C} 6$ position of sanguinarine against the nucleophilic attack by the hydroxide anion. ${ }^{18} \mathrm{~A}$ similar kind of binding affinity (iminium form encapsulation) has also been observed in the cyclodextrin nano-cavity and this binding is found to be entropy driven due to the release of water molecules from the cyclodextrin cavity. ${ }^{24}$ In contrast to previously reported literature, our work shows that different forms (iminium and alkanolamine) of SANG can be stabilized in different micellar environments. However, this feature is found to be absent in other drug delivery vehicles, like cyclodextrin and cucurbituril, which have selective preference for the iminium form over the alkanolamine form. ${ }^{18,24}$ Moreover, loading of this drug can be easily monitored with the help of fluorescence switch from orange (iminium) to violet (alkanolamine) without pursuing any sophisticated or complex technique, which may have significant application in the selective transportation of the drug in its active sites. As biomolecules have different affinities towards these two active forms of SANG (iminium and alkanolamine), we believe that our results may provide some new insight into the transportation of a particular form of SANG to its target site using micelles as drug delivery vehicles.

\section{Conclusion}

In this article, we have attempted to endeavor the physiochemical modulation of a potential anticancer drug, sanguinarine (SANG), inside different surface charged micellar arrangements. In CTAB (positively charged) and OBG (neutral) micelles, conversion from the iminium to alkanolamine form is confirmed from their respective changes in absorption and fluorescence spectra. The emission maximum at $580 \mathrm{~nm}$ (for the iminium form) is shifted to the characteristic alkanolamine peak at $420 \mathrm{~nm}$ in the abovementioned two micellar environments, and this conversion process is attributed to the decreased $\mathrm{p} K_{\mathrm{a}}$ of the SANG molecule inside these micelles. However, in the case of the negatively charged SDS micelle, the iminium form gets stabilized at the stern layer of the micelle due to the electrostatic attraction between positively charged iminium and negatively charged surfactant head groups. Time-resolved lifetime and anisotropy studies also upheld these facts and nicely compliment our steady state results in different micelles. Keeping the enormous 
biological applications of SANG in mind, our photophysical studies of SANG in bio-mimicking organized assemblies (i.e. micelles) may be helpful towards understanding their potential therapeutic applications.

\section{Acknowledgements}

Authors thank IISER-Pune for providing excellent experimental facilities. The authors are thankful to anonymous reviewers for their valuable comments and suggestions.

\section{References}

1 V. M. Dembitsky, T. A. Gloriozova and V. V. Poroikov, Phytomedicine, 2015, 22, 183-202.

2 M. Reina and A. González-Coloma, Phytochem. Rev., 2007, 6, 81-95.

3 G. B. Mahady and C. W. W. Beecher, Planta Med., 1994, 60, 553-557.

4 N. Ahmad, S. Gupta, M. M. Husain, K. M. Heiskanen and H. Mukhtar, Clin. Cancer Res., 2000, 6, 1524-1528.

5 I. D. Mandel, J. Am. Dent. Assoc., JADA, 1994, 125, 2S-10S.

6 I. De Stefano, G. Raspaglio, G. F. Zannoni, D. Travaglia, M. G. Prisco, M. Mosca, C. Ferlini, G. Scambia and D. Gallo, Biochem. Pharmacol., 2009, 78, 1374-1381.

7 V. M. Adhami, M. H. Aziz, S. R. Reagan-Shaw, M. Nihal, H. Mukhtar and N. Ahmad, Mol. Cancer Ther., 2004, 3, 933-940.

8 A. R. Hussain, N. A. Al-Jomah, A. K. Siraj, P. Manogaran, K. Al-Hussein, J. Abubaker, L. C. Platanias, K. S. Al-Kuraya and S. Uddin, Cancer Res., 2007, 67, 3888-3897.

9 J. Holy, G. Lamont and E. Perkins, BMC Cell Biol., 2006, 7, 1-12.

10 I. Bessi, C. Bazzicalupi, C. Richter, H. R. A. Jonker, K. Saxena, C. Sissi, M. Chioccioli, S. Bianco, A. R. Bilia, H. Schwalbe and P. Gratteri, ACS Chem. Biol., 2012, 7, 1109-1119.

11 P. Giri and G. S. Kumar, Biochim. Biophys. Acta, Gen. Subj., 2007, 1770, 1419-1426.

12 A. Adhikari, M. Hossain, M. Maiti and G. Suresh Kumar, J. Mol. Struct., 2008, 889, 54-63.

13 L.-P. Bai, Z. Cai, Z.-Z. Zhao, K. Nakatani and Z.-H. Jiang, Anal. Bioanal. Chem., 2008, 392, 709-716.

14 J. Urbanová, P. Lubal, I. Slaninová, E. Táborská and P. Táborský, Anal. Bioanal. Chem., 2009, 394, 997-1002.

15 M. Hossain, A. Y. Khan and G. Suresh Kumar, PLoS One, 2011, 6, e18333.

16 M. Hossain, A. Kabir and G. Suresh Kumar, J. Biomol. Struct. Dyn., 2012, 30, 223-234.

17 M. Maiti, R. Nandi and K. Chaudhuri, Photochem. Photobiol., 1983, 38, 245-249.

18 Z. Miskolczy, M. Megyesi, G. Tarkanyi, R. Mizsei and L. Biczók, Org. Biomol. Chem., 2011, 9, 1061-1070.

19 J. í. Dostál and M. Potáček, Collect. Czech. Chem. Commun., 1990, 55, 2840-2873.
20 A. Sen and M. Maiti, Biochem. Pharmacol., 1994, 48, 2097-2102.

21 M. Hossain, A. Y. Khan and G. Suresh Kumar, J. Chem. Thermodyn., 2012, 47, 90-99.

22 F. Wu, Y. Sun, Y. Shao, S. Xu, G. Liu, J. Peng and L. Liu, PLoS One, 2012, 7, e48251.

23 S. Hazra and G. Suresh Kumar, J. Phys. Chem. B, 2014, 118, 3771-3784.

24 S. Hazra and G. S. Kumar, RSC Adv., 2015, 5, 1873-1882.

25 K. Kataoka, A. Harada and Y. Nagasaki, Adv. Drug Delivery Rev., 2001, 47, 113-131.

26 M. L. Turco Liveri, M. Licciardi, L. Sciascia, G. Giammona and G. Cavallaro, J. Phys. Chem. B, 2012, 116, 5037-5046.

27 N. J. Turro, J. K. Barton and D. A. Tomalia, Acc. Chem. Res., 1991, 24, 332-340.

28 R. Chakrabarty, P. S. Mukherjee and P. J. Stang, Chem. Rev., 2011, 111, 6810-6918.

29 R. Villalonga, R. Cao and A. Fragoso, Chem. Rev., 2007, 107, 3088-3116.

30 C. Ghatak, V. G. Rao, S. Mandal, S. Ghosh and N. Sarkar, J. Phys. Chem. B, 2012, 116, 3369-3379.

31 S. Mandal, V. G. Rao, C. Ghatak, R. Pramanik, S. Sarkar and N. Sarkar, J. Phys. Chem. B, 2011, 115, 12108-12119.

32 H. C. Junqueira, D. Severino, L. G. Dias, M. S. Gugliotti and M. S. Baptista, Phys. Chem. Chem. Phys., 2002, 4, 2320-2328.

33 B. Bangar Raju and S. M. B. Costa, Phys. Chem. Chem. Phys., 1999, 1, 5029-5034.

34 B. Maity, A. Chatterjee, S. A. Ahmed and D. Seth, J. Phys. Chem. B, 2015, 119, 3776-3785.

35 C. C. Bof Bufon, J. D. Cojal González, D. J. Thurmer, D. Grimm, M. Bauer and O. G. Schmidt, Nano Lett., 2010, 10, 2506-2510.

36 M. Boncheva, D. H. Gracias, H. O. Jacobs and G. M. Whitesides, Proc. Natl. Acad. Sci. U. S. A., 2002, 99, 4937-4940.

37 K. M. Gangotri, R. C. Meena and R. Meena, J. Photochem. Photobiol., A, 1999, 123, 93-97.

38 A. Sengupta, R. V. Khade and P. Hazra, J. Phys. Chem. A, 2011, 115, 10398-10407.

39 K. Gavvala, W. D. Sasikala, A. Sengupta, S. A. Dalvi, A. Mukherjee and P. Hazra, Phys. Chem. Chem. Phys, 2013, 15, 330-340.

40 I. G. Motevich, N. D. Strekal, J. W. Nowicky and S. A. Maskevich, J. Appl. Spectrosc., 2010, 77, 394-399.

41 N. Sarkar, K. Das, A. Datta, S. Das and K. Bhattacharyya, J. Phys. Chem., 1996, 100, 10523-10527.

42 N. Nandi, K. Bhattacharyya and B. Bagchi, Chem. Rev., 2000, 100, 2013-2046.

43 V. E. Yushmanov, J. R. Perussi, H. Imasato, A. C. Ruggiero and M. Tabak, Biophys. Chem., 1994, 52, 157-163.

44 M. S. Fernandez and P. Fromherz, J. Phys. Chem., 1977, 81, 1755-1761.

45 D. Roy, R. Karmakar, S. K. Mondal, K. Sahu and K. Bhattacharyya, Chem. Phys. Lett., 2004, 399, 147-151. 
46 J. C. Koziar and D. O. Cowan, Acc. Chem. Res., 1978, 11, 50 J. R. Lakowicz, Principles of Fluorescence Spectroscopy, 334-341. Springer, New York, 3rd edn, 2006.

47 S. P. McGlynn, M. J. Reynolds, G. W. Daigre and N. D. 51 G. Duplâtre, M. F. Ferreira Marques and M. da Graça Christodoyleas, J. Phys. Chem., 1962, 66, 2499-2505. Miguel, J. Phys. Chem., 1996, 100, 16608-16612.

48 T. Wolff, Ber. Bunsenges. Phys. Chem., 1982, 86, 52 B. K. Paul, D. Ray and N. Guchhait, J. Phys. Chem. B, 2012, 1132-1134. 116, 9704-9717.

49 V. Ramesh and V. Ramamurthy, J. Photochem., 1984, 24, 53 B. Lorber, J. B. Bishop and L. J. DeLucas, Biochim. Biophys. 395-402. Acta, Biomembr., 1990, 1023, 254-265. 